\title{
THE POTENTIAL INFLUENCE OF SOIL SPLASHING BY RAINDROPS ON SEEDLING SURVIVAL ${ }^{1}$
}

\author{
BY JOHN R. CLEMENTS ${ }^{2}$
}

Soil splashing may be of interest to foresters because of its potential influence on seeds and seedlings especially on exposed mineral soil. This note discusses some observations made in a study of germination and survival of eastern white pine (Pinus strobus L.) in northern Michigan (Clements, 1963).

The impact of raindrops on exposed mineral soil splashes soil particles in the air and if vegetation is nearby the particles may be deposited on the plant surfaces. Osborn (1955) stated that the amount of soil splashed by a single drop is directly proportional to the square of the velocity of the drop and pointed out that as much as 700 pounds of soil per ton of oven-dry vegetation was left on forage after rains in some badly overgrazed pastures. The mechanism of the soil splash process was described by McIntyre (1958).

In the northern Michigan study continuous splashing during rain storms washed an undetermined number of seeds from scarified plots confusing somewhat the results of the experiment. Although differences between seedbed treatments were clear, statistical testing of the results was impossible. From an experimenter's point of view, even more serious is the possibility that seed removal from a plot may be greater under forest canopies or at the edge of forest clearings than in the open. Chapman (1948) estimated that for rainfall rates up to 2 inches per hour, which was slightly higher than his observed maximum rate, the kinetic energy of rainfall for each inch of rain per square foot of soil is considerably greater under a red pine canopy than in the open. Under the canopy the drops showed a greater range in size. Therefore direct comparison of germination data collected from plots under such conditions could be misleading.

Another influence of soil splashing seems beneficial. Soil particles adhered to the seed coats following rains, sometimes completely covering the seed. Besides reducing visibility to birds and rodents and lowering the maximum seed temperatures in the sun, the cemented particles provided anchorage permitting the elongating root tips to penetrate the soil surface. Some germinating seeds not so anchored died because the primary root could not penetrate the soil. The primary roots grew to half an inch or more, spiralling on top of the soil.

The most obvious potential influence is on survival of seedlings. Columns of soil were observed around the stems of young white pine seedlings growing on exposed mineral soil (Figure 1). These columns were built up around

\footnotetext{
${ }^{2}$ Department of Forestry, Canada, Forest Research Branch Contribution No. 621.

- Research Officer, Department of Forestry, Petawawa Forest Experiment Station, Chalk River, Ontaria.
} 
the stems during rain storms and adhered for several days, sometimes as much as a week or 10 days. Their duration appeared to depend on their dryness and on the amount of wind movement of the seedlings.

Such columns protect young seedlings from mortality due to lethal temperatures in direct sunlight. Solar heat would be dispersed by re-radiation to the surroundings, conduction down the column, and by evaporation of moisture from the soil column. The relative importance of the precise mechanisms of heat dispersion are not known.

The phenomena described here are doubtless more important than has hitherto been realized. They were observed on all scarified plots in the study and no doubt are common in nursery soils and other studies where mineral soil is exposed. As yet no quantitative information is available to assess their importance.

\section{REFERENCES}

CHAPMAN, G. 1948. Size of raindrops and their striking force at the soil surface in a red pine plantation. Trans. Amer. Geophys. Union 29: 664-670.

CLEMENTS, J. R, 1963. The influence of microrelicf on the establishment of coniferous reproduction. The University of Michigan, Doctoral Dissertation, Unpublished, $141 \mathrm{pp}$.

MeINTYRE, D. S. 1958. Soil splash and the formation of surface crusts by raindrop impact. Soil Sc. 85: 261-266.

OSBORN, B. 1955. How rainfall and runoff erode soil. Water, The Yearbook of Agriculture 1955, U.S. Dept. Agric. pp. 126-135.

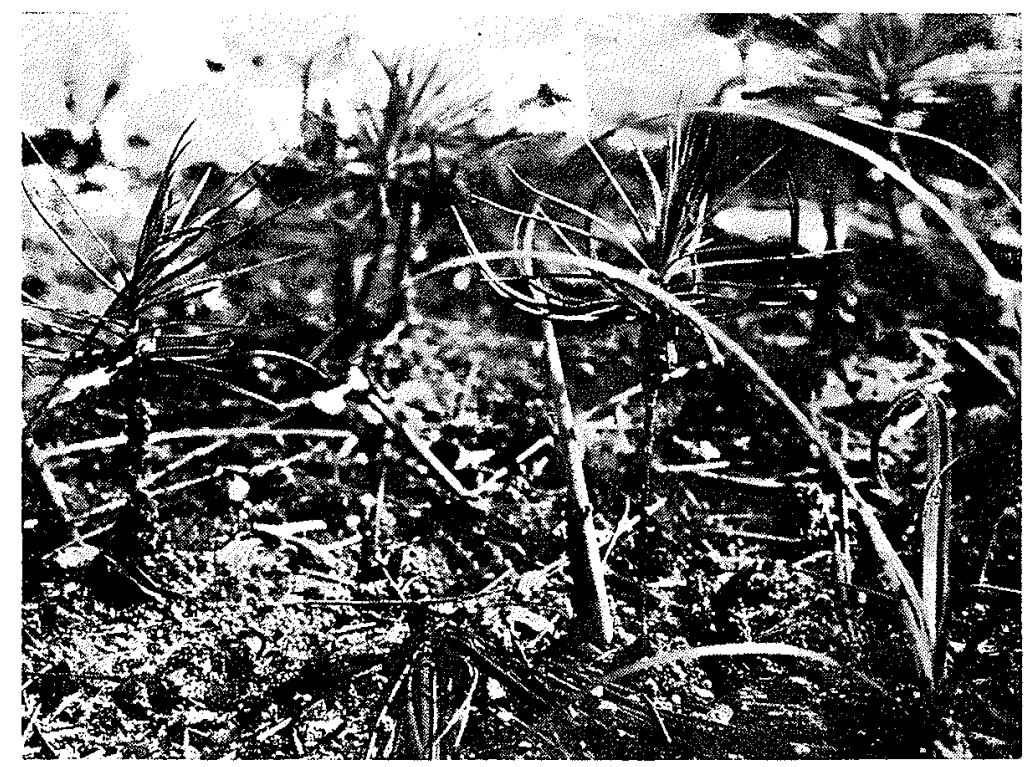

FIGURE 1. Soil columns on the stcms of young white pine scedlings causcd by rain spiash. They brak down with drying and wind movement, but usually last a week or 10 days. Toothpicks mark the location of the seedlings. 


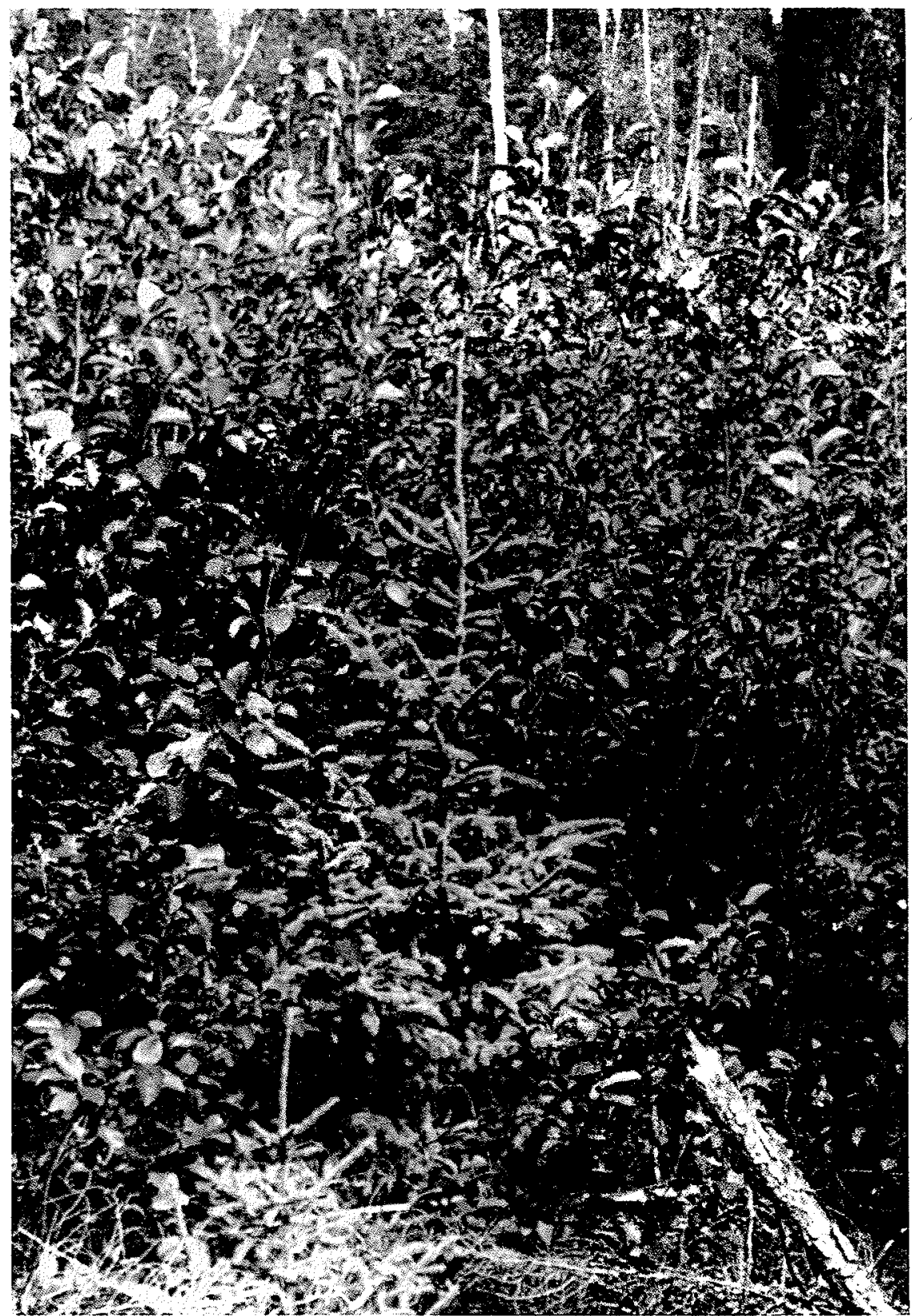

FIGURE 1. Two vigorous black spruce secdlings at the edge of an opening in a vigorous alder stand. Area cut cight years previously. 